\title{
Extraintestinal Crohn's Disease Mimicking Autoimmune Inner Ear Disease: A Histopathological Approach
}

\author{
Dettmer, M ; Hegemann, I ; Hegemann, S C A
}

\begin{abstract}
Patients with autoimmune inner ear disease develop rapidly progressive sensorineural hearing loss over a period of several weeks or months, often accompanied by vestibular loss. This disease can occur as a distinct clinical entity or in association with an underlying autoimmune disorder. Treatment comprises immunosuppression by corticosteroids, cytostatic drugs or tumor necrosis factor-alpha antagonists. We report histopathological and immunohistochemical findings of the inner ear of a patient with a granulomatous inner ear disease suffering from Crohn's disease that was nonresponsive to treatment and who underwent surgery for bilateral cochlear implants.
\end{abstract}

DOI: https://doi.org/10.1159/000315063

Posted at the Zurich Open Repository and Archive, University of Zurich ZORA URL: https://doi.org/10.5167/uzh-34825

Journal Article

Originally published at:

Dettmer, M; Hegemann, I; Hegemann, S C A (2010). Extraintestinal Crohn's Disease Mimicking Autoimmune Inner Ear Disease: A Histopathological Approach. Audiology and Neurotology, 16(1):36-40. DOI: https://doi.org/10.1159/000315063 
Autoimmune inner ear disease (AIED) as a manifestation of extraintestinal

Crohn's disease. A histopathological approach.

Authors:

Dettmer M., Hegemann I., Hegemann S. C. A.

Affiliation:

Zurich University Hospital

1) Institute of Surgical Pathology

2) Department of Internal Medicine, Division of Haematology

3) Department of Otorhinolaryngology, Head \& Neck Surgery

Running head

Crohn's disease in the inner ear

Address for correspondence:

Dr. Matthias Dettmer

University Hospital Zurich

Institute of Surgical Pathology

Schmelzbergstrasse 12

8091 Zurich

Phone: +41 (0)44 2552776

Fax: +41(0)442554416

e-mail: matthias.dettmer@usz.ch 


\begin{abstract}
Patients with autoimmune inner ear disease develop rapidly progressive sensorioneural hearing loss over a period of several weeks or months often accompanied by vestibular loss. This disease can appear as a distinct clinical entity or in association with an underlying autoimune disorder.

The treatment comprises immunosuppression corticosteroids, cytostatic drugs or TNF-alphaantagonists. We report here the histopathological and immunohistochemical findings of the inner ear in a patient with AIED and underlying Crohn's disease being non-responsive to any treatment and who underwent surgery for bilateral cochlear implants.
\end{abstract}

\title{
Case report:
}

A 27 year old teacher was assigned to our ENT outpatient department because of repetitive attacks of rotatory vertigo lasting about one day and repetitive sudden sensorineural hearing loss (SNHL). An ulcerous colitis was first diagnosed 9 month before (2006) and the patient was on oral prednisone $(20 \mathrm{mg} / \mathrm{d})$ and Imurek (150 mg/day). Tapering prednisone has been tried several times without success as bowel symptoms aggravated. Immunomodulatory therapy with infliximab was stopped because of side effects. In his family history were no autoimmune diseases or hearing losses. With normal ophthalmologic investigation Cogan's syndrome was ruled out for differential diagnosis. In June 2008 the patient developed aphtous lesions on the enoral mucosa and a granulomatous cheilitis. Because of that and an additionally occurring fistula of the colon with an abscess, the initial diagnosis of ulcerous colitis was changed to Crohn's disease.

The first vertigo attack was noticed about 6 weeks, and the first hearing loss about 3 weeks before we saw the patient. Hearing loss first occurred on the right ear with tinnitus. The patient had been treated outside with antibiotics for an otitis media despite lacking pain or pressure feeling in the ear. An audiogram was not available. The first audiogram in our clinic showed a bilateral SNHL of 50 to $55 \mathrm{db}$ at 0.125 to $2 \mathrm{kHz}, 90-100 \mathrm{db}$ at $3-8 \mathrm{kHz}$. On the left there was also SNHL of 20-35db between 0.125 and $4 \mathrm{kHz}$ and $45 / 35 \mathrm{db}$ at $6 / 8 \mathrm{kHz}$. At 12 $\mathrm{kHz}$ no tone was heard bilaterally. An MRI from Jan $15^{\text {th }} 2007$ showed a clear enhancement of contrast medium (CM, i.e.Gadolinium) in the complete left labyrinth. On April $24^{\text {th }} 2008$ there was still a significant CM-enhancement on the left and a possible enhancement in the semicircular canals on the right as well (Fig 1). 


\section{$\underline{\text { Figure } 1 \text { about here }}$}

The pure tone average (PTA) at $0.5,1$ and $4 \mathrm{kHz}$ was $26.25 \mathrm{db}$ on the left and $62.5 \mathrm{db}$ on the right ear. At that time the subjective visual vertical (SVV) was borderline tilted to the left (2 deg) and the caloric response was only $2 \%$ with warm water $\left(44^{\circ} \mathrm{C}\right)$ on the left and cold water $\left(30^{\circ} \mathrm{C}\right)$ on the right. He had a spontaneous nystagmus to the right of first degree according to Alexander at right gaze with a slow phase velocity of $2 \% \mathrm{~s}$ and complete visual suppression. There was also a head shaking nystagmus to the right of $3 \%$ s. Smooth pursuit and saccades were completely normal. There were no other neurological symptoms or signs. Vestibular evoked myogenic potentials (VEMPs) were reproducible on the right with clearly prolonged latencies ( $\mathrm{P}_{13}$ at 22.0 and $22.9 \mathrm{~ms}$ ) but could not be evoked on the left side. As half a year later there was no SPN, gaze evoked or head shaking nystagmus and only a caloric nystagmus of $2^{\circ}$ at cold water irrigation on the left and the head impulse test, measured with search coils showed gain values for all semicircular canal planes between 0.06 and 0.25 . A complete bilateral vestibular loss was diagnosed. Two transtympanic injections of about $0.5 \mathrm{ml}$ dexamethasone $(4 \mathrm{mg} / \mathrm{ml})$ in both ears had partly a short time but no longer lasting effect on hearing loss. Hearing was fluctuating with hearing losses every 3 to 7 days. 4-tone PTA was increasing from $62.5 / 26.25 \mathrm{db}$ (right/left) on August $28^{\text {th }} 2008$ to $113.7 \mathrm{db}$ on the right and complete deafness on the left on May $28^{\text {th }} 2009$.

Having several sudden sensorineural hearing losses on both sides the patient developed a bilateral complete labyrinthine lesion in less than 9 months with complete deafness and vestibular loss. Dexamethasone was given orally (40 mg/d and $10 \mathrm{mg} / \mathrm{d}$ for 3 days each) with no effect.

Summarizing clinical symptoms lead to the postulation of an autoimmune inner ear disease (AIED). 68 kD Western blot, previously related to AIED [Gottschlich et al., 1995; Moscicki et al., 1994] was not performed because sensitivity and specificity are greatly controversial. Laboratory tests revealed normal values for CRP at repetitive analysis. Antiviral IgG antibodies against herpes simplex 1(HSV1) and varicella zoster (VZV) were positive with negative IgM, implying a past infection. IgG against HSV2, borrelia burgdorferi and treponema pallidum were completely negative. Total serum IgG was borderline - $16.2 \mathrm{~g} / \mathrm{l}$ (normal value $<16.0 \mathrm{~g} / 1$ ). Antinuclear antibodies (ANA) and anti native DNA antibodies were negative whereas anti neutrophil cytoplasmatic antibodies (ANCA) were positive in low titer 1:40. Further specification showed neither classical reactivity to Proteinase 3 (PR3) nor atypical to myeloperoxidase (MPO). 
Furthermore, testing for antiphospholipid antibodies, anti-Cardiolipin and anti- $\beta 2$ -

Glycoprotein 1 revealed negative results for all Immunoglobulin classes as well as antimitochondrial and anti-smooth muscle antibodies.

A lumbar puncture was also performed with normal values for glucose, lactate and protein and $<1$ cell $/ \mu 1$.

After oral and intratympanic treatment with Dexamethasone was unsuccessfull, we started a treatment with infliximab (Remicade, $5 \mathrm{mg} / \mathrm{kg}$ ) intravenously. Albeit slight improvement on gastrointestinal symptoms, there was no significant effect on hearing and an MRI showed persistent $\mathrm{CM}$ enhancement in the labyrinths. Because of an occurring skin rush, the regimen was changed to Certolizimab (Cimzia, $400 \mathrm{mg}$ subcutaneously) which caused no side effects but neither improved nor stabilized hearing. Finally the patient got cochlear implants, first on the left and only 2 month later on the right side. On patients consent, we removed some tissue of the left ear for histopathological and immunohistochemical staining.

Histipathologic and immunopathologic results:

Conventional histology by HE staining showed mild chronic inflamed tissue with formation of granulomas (Fig. 2). The majority of the cellular content was identified as macrophages by CD 68 and CD 163 reactivity (Fig. 3).

Also some CD3 positive T lymphocytes were detected. Further substaining revealed some CD8 reactivity and absence of CD4 staining. (Fig. 4). Furthermore very few CD 20 positive mature B lymphocytes were detected, as well as some CD 138 positive plasma cells and very few CD 117 positive mast cells. There was a strong heme-oxygenase-1 (HO-1) expression (Fig. 5) elucidating inflammatory reaction and a strong positivity of the anti-apoptotic nuclear transcription factor kappa B (NFkappaB) (Fig. 6) which mediates at least partly HO-1 increase.

Figure 2-6 about here 


\section{Discussion}

Autoimmune inner ear disease (AIED) is a rare cause of sensorineural hearing loss which first was described in 1979 [McCabe, 1979]. It is important to recognise this disease entity, because early diagnosis and proper management may prevent complete hearing loss. The problem is, that there are only limited serologic tests available for the diagnosis of systemic autoimmune diseases [Dayal et al., 2008].

The pathogenesis of systemic autoimmune diseases remains unclear but antibodies directed against the inner ear and/or cellular effectors have been proposed [Staecker and Lefebvre, 2002; Veldman, 1998]. Among the candidates being associated with progressive sensorineural hearing loss are autoantigens against type II collagens, type IX collagens, $30 \mathrm{kD}$ proteins of inner ear membranes, laminin, $68 \mathrm{kD}$ proteins of inner ear, PO protein, Raf I protein and betatubulin [Yoo et al., 2002].

It can also occur as one of the clinical features of various systemic immune-mediated disorders, such as Cogan's syndrome, Behcet's disease, Wegener's granulomatosis, systemic sclerosis, systemic lupus erythematosus, giant cell arteritis, panarteritis nodosa, unclassified systemic vasculitis. Recently it has been linked to inflammatory bowel disease (IBD)[Karmody et al., 2009; Staecker and Lefebvre, 2002]. As distinct clinical entity without other immune-mediated disorders, it was termed AIED but nowadays, this term is also used if a typical inner ear disease is coupled to other autoimmune diseases. Being associated with so many different systemic disorders, it is not surprising that the clinical course is extremely variable. In contrast, if AIED is not associated to another autoimmune disorder, it is usually characterized by a rapidly developing (weeks or months) progressive hearing loss [Berrettini et al., 1998].

AIED is typically first treated with high-dose glucocorticoids [Dayal et al., 2008; Harris et al., 2003]. If this treatment is insufficient, other drugs like Methotrexate may be admistered although, some patients may still have progressive disease [Salley et al., 2001]. A newer promising treatment option is the administration of a tumor necrosis factor alpha blocker like Infliximab [Staecker and Lefebvre, 2002; Van Wijk et al., 2006].

Since the administration of different medications had no effect in our patient cochlear implants were applied. We were able to examine some tissue from his inner ear which was removed when cochlear electrodes were implanted. The tissue studied was situated in direct contact to the round window membrane and behind it. Histology revealed tissue with poor formed granulomas and abundant activated macrophages, being positive for CD68 and 
CD163 and strongly expressing HO-1. Beside that, there was an infiltrate of mononuclear cells with a strong activation of NFkappaB. Since our patient had Crohn's disease, it is possible that the granulomas we found in his inner ear are associated with this autoimmune disorder, since they are one of the histological hallmarks of Crohn's disease. There is evidence, that HO-1 is also involved in this complex autoimmune process in inflammatory bowel disease (IBD) and provides endogenous anti-oxidant and anti-inflammatory moieties which can modulate colonic inflammation [Horvath et al., 2008; Paul et al., 2005].

The effector immune cells produce high levels of pro-inflammatory cytokines in IBD and the nuclear transcription factor kappaB pathway was identified as one of the key factors in this scenario [Atreya et al., 2008; Ellis et al., 1998].

Crohn's disease is considered a systemic immunopathy which has many extraintestinal well documented manifestations like joints, eyes or other regions from the head like the larynx, oral cavity and the nose [Aloi and Cucchiara, 2009; Bradley et al., 2004; Lakatos et al., 2003]. According to our knowledge, this is the first reported histopathological and immunhistological approach to AIED. The described entities resemble histological finings in Crohns disease. Therefore, we suggest, that the described case of AIED is not a disease entity of its own but may be an extraintestinal manifestation of Crohn's disease. The many varying clinical courses of AIED may reflect different activation stages of differing underlying autoimmune disorders.

As in Crohn's disease, not all patients can be successfully treated with standard immunosuppression and also some patients with AIED may require the new tumor necrosis factor alpha blockers for beneficial treatment [Rutgeerts et al., 2009; Staecker and Lefebvre, 2002; Van Wijk et al., 2006]. But since not all patients with AIED, like ours, can be treated successfully with the current available medications, there is need for new treatment options and better characterization of the underlying immunoprocess. 


\section{References}

Aloi M, Cucchiara S: Extradigestive manifestations of ibd in pediatrics. Eur Rev Med Pharmacol Sci 2009;13 Suppl 1:23-32.

Atreya I, Atreya R, Neurath MF: Nf-kappab in inflammatory bowel disease. J Intern Med 2008;263:591-596.

Berrettini S, Ravecca F, Bruschini L, Ursino F, Sellari-Franceschini S: [progressive sensorineural hearing loss: Immunologic etiology]. Acta Otorhinolaryngol Ital 1998;18:33-41.

Bradley PJ, Ferlito A, Devaney KO, Rinaldo A: Crohn's disease manifesting in the head and neck. Acta Otolaryngol 2004;124:237-241.

Dayal VS, Ellman M, Sweiss N: Autoimmune inner ear disease: Clinical and laboratory findings and treatment outcome. J Otolaryngol Head Neck Surg 2008;37:591-596.

Ellis RD, Goodlad JR, Limb GA, Powell JJ, Thompson RP, Punchard NA: Activation of nuclear factor kappa $\mathrm{b}$ in crohn's disease. Inflamm Res 1998;47:440-445.

Gottschlich S, Billings PB, Keithley EM, Weisman MH, Harris JP: Assessment of serum antibodies in patients with rapidly progressive sensorineural hearing loss and meniere's disease. Laryngoscope 1995;105:1347-1352.

Harris JP, Weisman MH, Derebery JM, Espeland MA, Gantz BJ, Gulya AJ, Hammerschlag PE, Hannley M, Hughes GB, Moscicki R, Nelson RA, Niparko JK, Rauch SD, Telian SA, Brookhouser PE: Treatment of corticosteroid-responsive autoimmune inner ear disease with methotrexate: A randomized controlled trial. JAMA 2003;290:18751883.

Horvath K, Varga C, Berko A, Posa A, Laszlo F, Whittle BJ: The involvement of heme oxygenase-1 activity in the therapeutic actions of 5-aminosalicylic acid in rat colitis. Eur J Pharmacol 2008;581:315-323.

Karmody CS, Valdez TA, Desai U, Blevins NH: Sensorineural hearing loss in patients with inflammatory bowel disease. Am J Otolaryngol 2009;30:166-170.

Lakatos L, Pandur T, David G, Balogh Z, Kuronya P, Tollas A, Lakatos PL: Association of extraintestinal manifestations of inflammatory bowel disease in a province of western hungary with disease phenotype: Results of a 25-year follow-up study. World J Gastroenterol 2003;9:2300-2307.

McCabe B: Autoimmune sensorineural hearing loss. . Ann Otol Rhinol Laryngol 1979;5:585589.

Moscicki RA, San Martin JE, Quintero CH, Rauch SD, Nadol JB, Jr., Bloch KJ: Serum antibody to inner ear proteins in patients with progressive hearing loss. Correlation with disease activity and response to corticosteroid treatment. JAMA 1994;272:611616.

Paul G, Bataille F, Obermeier F, Bock J, Klebl F, Strauch U, Lochbaum D, Rummele P, Farkas S, Scholmerich J, Fleck M, Rogler G, Herfarth H: Analysis of intestinal haemoxygenase-1 (ho-1) in clinical and experimental colitis. Clin Exp Immunol 2005; 140:547-555.

Rutgeerts P, Vermeire S, Van Assche G: Biological therapies for inflammatory bowel diseases. Gastroenterology 2009;136:1182-1197.

Salley LH, Jr., Grimm M, Sismanis A, Spencer RF, Wise CM: Methotrexate in the management of immune mediated cochleovesitibular disorders: Clinical experience with 53 patients. J Rheumatol 2001;28:1037-1040.

Staecker H, Lefebvre PP: Autoimmune sensorineural hearing loss improved by tumor necrosis factor-alpha blockade: A case report. Acta Otolaryngol 2002;122:684-687. 
Van Wijk F, Staecker H, Keithley E, Lefebvre PP: Local perfusion of the tumor necrosis factor alpha blocker infliximab to the inner ear improves autoimmune neurosensory hearing loss. Audiol Neurootol 2006;11:357-365.

Veldman J: Immune-mediated sensorineural hearing loss. Auris Nasus Larynx 1998;25:309317.

Yoo TJ, Du X, Kwon SS: Molecular mechanism of autoimmune hearing loss. Acta Otolaryngol Suppl 2002:3-9. 
Legends

Fig.1:

T1 weighted MRI with Gadolinium showing slight gadolinium-enhancement in the left cochlea (arrow)

Fig. 2:

Mild chronic inflamed tissue with formation of granulomas (magnification

X 100)

Fig. 3:

CD68 positive Macrophages are abundant and are arranged in poorly formed granulomas (magnification X 200)

Fig. 4:

Some T lymphocytes, expressing CD8 (magnification X 200)

Fig. 5:

Strong heme-oxygenase-1 (HO-1) expression in activated macrophages (magnification X 200)

Fig. 6:

Strong activation of the anti-apoptotic nuclear transcription factor kappa B (NFkappaB) (magnification X 200) 